\title{
THE LEVERAGE EFFECT AND THE ASYMMETRY OF THE ERROR DISTRIBUTION IN GARCH-BASED MODELS: THE CASE OF BRAZILIAN MARKET RELATED SERIES
}

\author{
Daniel de Almeida and Luiz K. Hotta*
}

Received September 22, 2012 / Accepted October 14, 2013

\begin{abstract}
Traditional GARCH models fail to explain at least two of the stylized facts found in financial series: the asymmetry of the distribution of errors and the leverage effect. The leverage effect stems from the fact that losses have a greater influence on future volatilities than do gains. Asymmetry means that the distribution of losses has a heavier tail than the distribution of gains. We test whether these features are present in some series related to the Brazilian market. To test for the presence of these features, the series were fitted by $\operatorname{GARCH}(1,1)$, TGARCH(1,1), $\operatorname{EGARCH}(1,1)$, and GJR-GARCH$(1,1)$ models with standardized Student $t$ distribution errors with and without asymmetry. Information criteria and statistical tests of the significance of the symmetry and leverage parameters are used to compare the models. The estimates of the VaR (value-at-risk) are also used in the comparison. The conclusion is that both stylized facts are present in some series, mostly simultaneously.
\end{abstract}

Keywords: asymmetry in volatility models, asymmetric Garch family models, VaR (Value-at-Risk).

\section{INTRODUCTION}

Two important features usually found in time series of asset returns are the presence of volatility clustering and the high kurtosis. Here volatility is considered as the conditional variance, although some authors define it as the conditional standard deviation. The most popular model used in the literature to explain these two stylized facts is the generalized autoregressive conditional heteroskedastic (GARCH) model of Bollerslev (1986) [3] with symmetric errors (normal or Student $t$ distributions). However, these traditional GARCH models cannot explain some stylized facts found in financial time series. Two important unexplained facts are the skewness, or asymmetry, in the distribution of the errors and the leverage effect. The former consists of losses having a distribution with a heavier tail than gains. Simkowitz \& Beedles (1980) [18], Kon (1984) [10], among others drew the attention to the skewness in such distribution. French et al. (1987)

*Corresponding author

University of Campinas, Rua Sérgio Buarque de Holanda, 651, Distr. Barão Geraldo, 13083-859 Campinas, SP, Brazil. Phone: +55(19) 3521-6081/93101120. E-mails: dani.d.almeida89@gmail.com; hotta@ime.unicamp.br. 
[8] found that the conditional asymmetry coefficient significantly differs from zero in the standardized residuals when ARCH family models were fitted to the daily returns of the Standard \& Poor 500 (S\&P) series. The leverage effect, originally introduced by Black (1976) [2], takes into account that losses have a greater influence on future volatility than do the gains. However, no study has tested yet for the simultaneous presence of these two effects, especially for Brazilian related series.

The aim of the present paper is to verify if these stylized facts are present in some market indices related to the Brazilian market and five of the most important stocks traded in the São Paulo Stock Exchange (BOVESPA). The indices considered are the Ibovespa (IBV, Brazil), MERVAL (Argentina), and S\&P (USA), and the five stocks are Itaú-Unibanco (Itaú), Vale PNA (Vale), Petrobrás PN (Petro), Banco do Brasil ON (BB), and Bradesco PN (Brad), in the period from February 1st, 2000 to February 1st, 2011. After filtering the return series with AR(1) models, we fitted the GARCH(1,1), TGARCH(1,1), EGARCH(1,1), and GJR-GARCH(1,1) models with standardized Student $t$ and standardized asymmetric Student $t$ innovations, for a total of eight models.

Three methods are used to compare the models. The first one uses the Akaike information criterion (AIC) (Akaike, 1974 [1]), the Bayesian information criterion (BIC) (Schwarz, 1978 [17]), and the Hannan and Quin information criterion (HQ) (Hannan \& Quinn, 1979 [11]), to select the best model. The second method tests the significance of the symmetry and leverage parameters. The third method compares the value at risk ( $\mathrm{VaR})$ estimated by the eight models treated. A model is considered adequate if the VaR estimates have the desired properties. Section 2 presents three GARCH family models which have leverage effect and the asymmetric distribution used to model the error term. Section 3 presents the methods used to compare these models and Section 4 presents some applications. Our concluding remarks are in Section 5.

\section{ARMA-GARCH MODELS}

Denoting the returns by $r_{t}$, this series is first filtered by an ARMA model (1), yielding residuals $\varepsilon_{t}$, serially uncorrelated, but not necessarily independent. In (2)-(4), the series $\varepsilon_{t}$ is fitted by a conditional volatility model. We can write this class of models as

$$
\begin{aligned}
r_{t} & =\mu_{t}+\varepsilon_{t} \\
\varepsilon_{t} & =\sigma_{t} z_{t} \\
\mu_{t} & =c\left(\mu \mid \Omega_{t-1}\right) \\
\sigma_{t}^{2} & =h\left(\mu, \eta \mid \Omega_{t-1}\right),
\end{aligned}
$$

where $c\left(. \mid \Omega_{t-1}\right)$ and $h\left(. \mid \Omega_{t-1}\right)$ are functions of $\Omega_{t-1}=\left\{r_{j}, j \leq t-1\right\}$, and $z_{t}$ is an independent and identically distributed (i.i.d.) process, independent of $\Omega_{t-1}$, with $\mathrm{E}\left(z_{t}\right)=0$ and $\operatorname{Var}\left(z_{t}\right)=1$. In the ARMA-GARCH model, the residuals are modeled by the generalized autoregressive conditional heteroskedasticity $(\mathrm{GARCH})$ model and the shapes of the functions $c\left(. \mid \Omega_{t-1}\right)$ and $h\left(. \mid \Omega_{t-1}\right)$ are defined by the orders of the ARMA and GARCH models, respectively. Assuming their existence, $\mu_{t}$ and $\sigma_{t}^{2}$ are the conditional mean and variance of $r_{t}$, respectively. 
For example, in the AR(1)-GARCH(1,1) model, the mean and the volatility given by (3) and (4), respectively, are

$$
\begin{aligned}
& \mu_{t}=\mu+\phi r_{t-1} \\
& \sigma_{t}^{2}=\omega+\alpha \varepsilon_{t-1}^{2}+\beta \sigma_{t-1}^{2},
\end{aligned}
$$

with $|\phi|<1$ and $\omega>0$. The conditions $\alpha, \beta \geq 0, \alpha+\beta<1$, which are sufficient conditions for the process to be stationary and have finite variance, therefore, are usually adopted.

\subsection{The leverage effect}

The leverage effect is caused by the fact that negative returns have a greater influence on future volatility than do positive returns. For a good comparison among several GARCH models with leverage effect, see Rodríguez \& Ruiz (2012) [16]. In this paper, we consider three of the most popular models to represent it: the EGARCH, TGARCH, and GJR models.

In the EGARCH model (Nelson, 1991 [15]), the conditional volatility is given by

$$
\ln \left(\sigma_{t}^{2}\right)=\omega+\gamma_{E} z_{t-1}+\alpha\left\{\left|z_{t-1}\right|-E\left(\left|z_{t-1}\right|\right)\right\}+\beta \ln \left(\sigma_{t-1}^{2}\right) .
$$

Since $z_{t}$ is an i.i.d. sequence, $\left|\varepsilon_{t}\right|-E\left(\left|\varepsilon_{t}\right|\right)$ is also a sequence of i.i.d. random variables with zero mean. $\gamma_{E}$ is a real parameter, such that $\gamma_{E}<0$ when negative returns have a greater impact on future volatility than positive returns. Due to the volatility specification in terms of the logarithmic transformation, there are no restrictions on the parameters to ensure positive variance. A sufficient condition for stationarity and finite kurtosis is $|\beta|<1$.

The Threshold GARCH (TGARCH)(see Zakoïan, 1994 [19]) is a particular case of a nonlinear ARCH model and it models the conditional standard deviation instead of the conditional variance. The TGARCH(1,1) is written as

$$
\sigma_{t}=\omega+\alpha\left|\varepsilon_{t-1}\right|+\beta \sigma_{t-1}+\gamma_{T} \varepsilon_{t-1}
$$

Ding et al. (1993) [5] proved that, in order to guarantee the positivity of $\sigma_{t}$, it is sufficient that $\omega>0, \alpha \geq 0$ and $\gamma_{T}<\alpha$. Furthermore, the model is stationarity if $\gamma_{T}^{2}<1-\alpha^{2}-\beta^{2}-$ $2 \alpha \beta E\left(\left|z_{t}\right|\right)$. For example, if $z_{t}$ is Gaussian, then $E\left(\left|z_{t}\right|\right)=\sqrt{\frac{2}{\pi}}$.

The GJR model of Glosten et al. (1993) [9] specifies the conditional variance by

$$
\sigma_{t}^{2}=\omega+\alpha \varepsilon_{t-1}^{2}+\beta \sigma_{t-1}^{2}+\gamma_{G} \mathbb{I}\left(z_{t-1}<0\right) \varepsilon_{t-1}^{2},
$$

where $\mathbb{I}($.$) is equal to 1$ when the inequality is satisfied and 0 otherwise. Hentschel (1995) [13] showed that $\sigma_{t}^{2}$ is positive if

$$
\omega>0, \alpha, \beta, \gamma_{G} \geq 0 .
$$

A sufficient condition for stationarity and finite variance is

$$
\gamma_{G}<2(1-\alpha-\beta) .
$$




\subsection{Asymmetry in the errors}

In practice, it is generally assumed that $z_{t} \sim N(0,1)$ or $z_{t} \sim t_{v}$ standardized, or any distribution that describes the heavy tails of financial time series. For normal errors and $\operatorname{GARCH}(1,1)$, the kurtosis is equal to

$$
K=\frac{\mathbb{E}\left(r_{t}^{4}\right)}{\left[\mathbb{E}\left(r_{t}^{2}\right)\right]^{2}}=\frac{3[1-(\alpha+\beta)]}{1-(\alpha+\beta)^{2}-2 \alpha_{1}^{2}}>3,
$$

when the fourth moment is defined, i.e., when the denominator is positive. This shows that even when the error $z_{t}$ has a standard normal distribution and $\varepsilon_{t}$ follows a GARCH process, the tails of $\varepsilon_{t}$ are heavier than normal. However, in empirical series it is often found that the distribution of the error term $z_{t}$ has heavier tails than the normal distribution, and is often replaced by the standardized Student $t$ distribution (see, for example, Bollerslev, 1986 [3]).

The standardized Student $t$ distribution with $v(v>2)$ degrees of freedom is given by

$$
g(z)=\frac{\Gamma\left(\frac{v+1}{2}\right)}{\sqrt{(v-2) \pi} \Gamma(v / 2)}\left(1+\frac{z^{2}}{(v-2)}\right)^{-\left(\frac{v+1}{2}\right)},
$$

where $\Gamma$ is the gamma function.

The distribution given in (13) has skewness coefficient equal to zero and the excess of kurtosis equal to $6 /(v-4)$, for $v>4$.

While the high kurtosis of returns is a well established fact, the situation is much more obscure for the symmetry of the distribution of $z_{t}$. In this paper, we consider the asymmetric Student $t$ distribution. There have been several proposals to include asymmetry in the Student $t$ distribution. Hansen (1994) [12] was the first to use an asymmetric Student $t$ distribution in modeling financial data. Fernández \& Steel (1998) [7] proposed a way of introducing asymmetry into any symmetric and unimodal continuous distribution $g($.), changing its scale on each side of the mode. Applying this procedure to the Student $t$ distribution, one obtains an asymmetric Student $t$ density. In order to preserve the specifications of the GARCH model, Lambert \& Laurent (2001) [14] modified this density to standardize it, that is, to have zero mean and unit variance.

Following Lambert \& Laurent (2001) [14], the random variable $z_{t}$ is said to follow the standardized asymmetric Student $t$, denoted by $\operatorname{SKST}(0,1, \xi, v)$, with parameters $v>2$ (the number of degrees of freedom) and $\xi>0$ (the parameter associated with the skewness), if its density is of the form

$$
f\left(z_{t} \mid \xi, v\right)= \begin{cases}\frac{2}{\xi+\frac{1}{\xi}} s g\left[\xi\left(s z_{t}+m\right) \mid v\right] & \text { if } \quad z_{t}<-m / s \\ \frac{2}{\xi+\frac{1}{\xi}} s g\left[\left(s z_{t}+m\right) / \xi \mid v\right] & \text { if } \quad z_{t} \geq-m / s\end{cases}
$$


where $g(. \mid v)$ is the density of the standardized symmetric Student $t$ given by (13), and the constants $m=m(\xi, v)$ and $s=\sqrt{s^{2}(\xi, v)}$ are, respectively, the mean and standard deviation of the $\operatorname{SKST}\left(m, s^{2}, \xi, v\right)$ distribution and can be expressed by

$$
m(\xi, v)=\frac{\Gamma\left(\frac{v+1}{2}\right) \sqrt{v-2}}{\sqrt{\pi} \Gamma\left(\frac{v}{2}\right)}\left(\xi-\frac{1}{\xi}\right)
$$

and

$$
s^{2}(\xi, v)=\left(\xi^{2}+\frac{1}{\xi^{2}}-1\right)-m^{2},
$$

respectively (Fernández \& Steel, 1998 [7]). The main advantages of this density are its easy implementation and the clear interpretation of its parameters. Ehlers (2012) [6] modeled GARCH model with the error term errors with this distribution and proposed a fully Bayesian approach to estimate the model.

\section{CRITERIA FOR COMPARISON OF MODELS}

Consider $T$ observations of a volatility process and suppose that we want to verify the presence of the leverage effect and of asymmetry in the perturbations. In order to do this, we use the following eight models: GARCH, TGARCH, EGARCH, and GJR-GARCH with standardized symmetric and asymmetric Student $t$ distributions. In this section, we present the three criteria used to select the most appropriate model.

Information criteria. There are several information criteria suggested in the literature to select a model. In this paper, we consider the AIC, BIC, and HQ criteria. These criteria are the likelihood penalized by different functions of the number of parameters of the model.

Testing hypotheses. By fitting the GJR-GARCH model with asymmetric Student $t$ distribution, for example, we have as special cases a model without leverage when $\gamma_{G}=0$ and a model with symmetric innovations when the skewness parameter $(\xi)$ is equal to 1 . Thus we can use hypothesis testing to verify the presence or absence of these two stylized facts. We can follow the same procedure with GARCH, TGARCH, and EGARCH models.

The third criterion uses the VaR at the $95 \%$ and $99 \%$ levels to test the accuracy of the models in making predictions. We use the conditional prediction interval evaluation procedure of Christoffersen (1998) [4]. He proposed a likelihood ratio (LR) test to test the null hypothesis that a statistical method (the model) is good for prediction purpose. This test is defined as follows.

\subsection{The likelihood ratio test for the conditional coverage}

The VaR can be viewed as a prediction interval. One of the methods to evaluate prediction interval is the LR test of Christoffersen (1998) [4]. In the VaR case, it tests whether the sequence of losses smaller than the VaR comes from a random sample of the Bernoulli distribution with probability equal to the nominal value. 
Let $\left(r_{t}\right)_{1 \leq t \leq T}$ be the realization of a series of returns of any financial asset and let $\left[L(p)_{t \mid t-1}\right.$, $U(p))_{t \mid t-1}$ ] be the corresponding sequence of interval forecast outside the sample, where $L(p)_{t \mid t-1}$ and $\left.U(p)\right)_{t \mid t-1}$ are the lower and upper limits of the forecast intervals at time $t$, given the information until time $t-1$, at the confidence level $p$. Set the indicator variable $I_{t}$ at time $t$, given information until time $t-1$, as

$$
I_{t}=\left\{\begin{array}{lll}
1, & \text { if } & \left.r_{t} \in\left[L(p)_{t \mid t-1}, U(p)\right)_{t \mid t-1}\right] \\
0, & \text { if } & r_{t} \notin\left[L(p)_{t \mid t-1}, U(p)_{t \mid t-1}\right] .
\end{array}\right.
$$

We say that the sequence of prediction interval, $\left.\left[L(p)_{t \mid t-1}, U(p)\right)_{t \mid t-1}\right]$, is efficient with respect to the information set at time $t-1\left(\Psi_{t-1}\right)$, if $E\left(I_{t} \mid \Psi_{t-1}\right)=p, \forall t$ if it passes the LR test. Christoffersen (1998) [4] showed that testing $E\left(I_{t} \mid \Psi_{t-1}\right)=p$, for all $t$, is equivalent to testing if the sequence $\left(I_{t}\right)_{1 \leq t \leq T}$ is i.i.d. with a Bernoulli distribution with parameter $p$, i.e., $I_{t} \sim$ i.i.d. Ber(p). Therefore, a sequence of prediction intervals, $\left.\left[L(p)_{t \mid t-1}, U(p)\right)_{t \mid t-1}\right]$, has a correct conditional coverage if $I_{t} \sim \operatorname{Ber}(\mathrm{p})$ i.i.d., $\forall t$.

In the conditional coverage test, the null hypothesis is that $\left(I_{t}\right)_{1 \leq t \leq T}$ is independent and $E\left(I_{t} \mid \Phi_{t-1}\right)=p$. The test statistics is

$$
\left.L R_{c c}=-l\left(p ; I_{1}, \ldots, I_{T}\right)-l\left(\hat{\pi}_{1} ; I_{1}, \ldots, I_{T}\right)\right],
$$

where $l\left(\theta ; ; I_{1}, \ldots, I_{T}\right)$ is the $\log$ likelihood function, i.e., $l\left(p ; I_{1}, \ldots, I_{T}\right)=\left(n_{T}\right) \log (\theta)+$ $\left(T-n_{T}\right) \log (1-\theta)$ with $n_{T}=\sum_{i=1}^{T} I_{i}$, and $\hat{\pi}_{1}=n_{T} / T$. The statistics $L R_{c c}$ has a $\chi_{2}^{2}$ distribution under the null hypothesis.

Equation (18) can be written as the sum of the LR test statistics for the correct unconditional coverage and the LR test statistics for independence (Christoffersen, 1998 [4]). Rejecting the null hypothesis implies that the model is not good for prediction purpose.

\section{APPLICATIONS}

In this section, we analyze the series of returns of IBV, Merval, S\&P, Itaú, Vale, Petro, BB, and Brad, from February 1st, 2000 to February 1st, 2011, with a total of 12 years. Each series was previously filtered by an $\operatorname{ARMA}(p, q)$ model with appropriate orders.

For each dataset we adopted the following procedure.

1. Consider the observations of the returns of the first eight years.

2. Fit all eight models.

3. Verify which model is selected by the AIC, BIC and HQ criteria.

4. For each estimated model, evaluate the one-step-ahead $95 \%$-VaR and $99 \%$-VaR for the next five days. Test whether the returns are below the estimated VaR values. Note that we are always doing one-step-ahead estimation of the VaR, but the model is not re-estimated every time we include one observation. 
5. Include five more observations and exclude the first five observations.

6. Repeat steps (2) to (5) until the end of the period.

For each series and each model, we fitted around 200 models and estimated around 1,000 VaR values. The number of models and VaR estimates depend upon each series, because we ignored non-trading days.

Tables 1 and 2 indicate how many times each of the eight models were selected by the AIC, BIC, and HQ criteria. The main conclusions are:

- The GARCH model was never selected by any criterion for the IBV, S\&P, Itaú, Petro, or Brad series. For the Merval and Vale series, the GARCH model was only selected by the BIC ( $60 \%$ of the time for the Merval series and $21 \%$ for the Vale series); for the BB series the GARCH model was only selected $31 \%$ of the time. This means that there is a clear preference of the information criteria for models with the leverage effect.

- For all of the stocks, the GJR was the most selected model by all the criteria. For the Petro and Brad series, it was always the model selected. For the Merval series, the GJR model was always selected by the AIC, in $91 \%$ of the cases by the HQ criterion, and $40 \%$ by the BIC. The TGARCH was selected most of the time for the IBV series by all criteria, and the EGARCH model was selected most of the time for the S\&P series by all criteria.

- For the IBV and S\&P series, the criteria selected models with leverage and asymmetric distributions almost all the time. For the Merval, Itaú, and Brad series, the criteria selected models with leverage and asymmetric distributions most of the time. For the Vale, Petro, and BB series, the criteria selected models with leverage and symmetric distributions most of the time.

Tables 3 and 4 present, respectively, the percentage of cases where the asymmetry and leverage parameters were significant at the $5 \%$ level. Figure 1 presents the estimated asymmetry and leverage parameters in the GJR-GARCH asymmetric model for the IBV, Merval, Vale, and BB series, while Figure 2 presents the results for the Itaú, S\&P, Petro, and Brad series. We do not present the equivalent graphs for the other models, since their behavior is very similar to that of the GJR model. Under the null hypothesis of no asymmetry, one has $\xi=1$; and under the null hypothesis of no leverage effect, $\gamma_{G}=0$. We use full symbols to indicate rejection of the null hypotheses at the $5 \%$ level. The main conclusions are:

- For the GJR model, the leverage effect was detected in the models with symmetric and asymmetric errors in all cases for the IBV, S\&P, Itaú, Petro, and Brad series, and in practically all cases for the Vale series. For the Merval and BB series, the leverage effect was detected in approximately $80 \%$ and $67 \%$ of the cases, respectively. The results were similar in the EGARCH and TGARCH models. 
Table 1 - Number of times the model was selected by the AIC, BIC, and HQ criteria. Panels 1-8 correspond to the IBV, Merval, S\&P, Itaú, Vale, Petro, BB, and Brad series, respectively. sym., asym. $=$ standardized symmetric and asymmetric Student $t$ innovations, respectively.

\begin{tabular}{|c|c|c|c|c|c|c|c|c|}
\hline \multirow{2}{*}{ Criterion } & \multicolumn{2}{|c|}{ GARCH } & \multicolumn{2}{|c|}{ GJR } & \multicolumn{2}{|c|}{ EGARCH } & \multicolumn{2}{|c|}{ TGARCH } \\
\hline & sym. & asym. & sym. & asym. & sym. & asym. & sym. & asym. \\
\hline AIC & 0 & 0 & 0 & 30 & 0 & 26 & 0 & 141 \\
\hline BIC & 0 & 0 & 0 & 30 & 8 & 18 & 3 & 138 \\
\hline HQ & 0 & 0 & 0 & 30 & 0 & 26 & 0 & 141 \\
\hline AIC & 0 & 0 & 20 & 176 & 0 & 0 & 0 & 0 \\
\hline BIC & 66 & 52 & 37 & 41 & 0 & 0 & 0 & 0 \\
\hline HQ & 0 & 17 & 46 & 133 & 0 & 0 & 0 & 0 \\
\hline AIC & 0 & 0 & 0 & 13 & 0 & 142 & 0 & 46 \\
\hline BIC & 0 & 0 & 0 & 13 & 22 & 120 & 0 & 46 \\
\hline HQ & 0 & 0 & 0 & 13 & 6 & 136 & 0 & 46 \\
\hline AIC & 0 & 0 & 0 & 92 & 0 & 40 & 0 & 65 \\
\hline BIC & 0 & 0 & 25 & 53 & 49 & 0 & 38 & 32 \\
\hline HQ & 0 & 0 & 9 & 81 & 10 & 30 & 6 & 61 \\
\hline AIC & 0 & 0 & 183 & 0 & 0 & 0 & 14 & 0 \\
\hline BIC & 42 & 0 & 141 & 0 & 0 & 0 & 14 & 0 \\
\hline HQ & 0 & 0 & 183 & 0 & 0 & 0 & 14 & 0 \\
\hline AIC & 0 & 0 & 38 & 159 & 0 & 0 & 0 & 0 \\
\hline BIC & 0 & 0 & 197 & 0 & 0 & 0 & 0 & 0 \\
\hline HQ & 0 & 0 & 152 & 45 & 0 & 0 & 0 & 0 \\
\hline AIC & 0 & 42 & 69 & 86 & 0 & 0 & 0 & 0 \\
\hline BIC & 81 & 0 & 116 & 0 & 0 & 0 & 0 & 0 \\
\hline HQ & 1 & 58 & 102 & 36 & 0 & 0 & 0 & 0 \\
\hline AIC & 0 & 0 & 12 & 185 & 0 & 0 & 0 & 0 \\
\hline BIC & 0 & 0 & 111 & 86 & 0 & 0 & 0 & 0 \\
\hline HQ & 0 & 0 & 62 & 135 & 0 & 0 & 0 & 0 \\
\hline
\end{tabular}

- The asymmetry in the errors was detected in all the cases for all models for the IBV and S\&P series, and in approximately 75\%,70\%, and 50\% of the cases for the Merval, Brad, and BB series, respectively. For the Vale series, the null hypothesis was never rejected. For the Itaú and Petro series, the percentage depended on the model. For the Itaú series, the detection of asymmetry varied from $99.5 \%$ for the GARCH model to $74.6 \%$ for the TGARCH model, while for the Petro series the percentages varied from $34.5 \%$ for the GJR model to $9.1 \%$ for the EGARCH model.

- From the figures we can observe that there is a certain stability in time and that in most of the cases the leverage effect and the asymmetry are simultaneously significant most of the time. 
Table 2 - Percentage of selection of a model with leverage (GJR, EGARCH, TGARCH) and without leverage (GARCH), and with and without asymmetric innovations. The left side panels 1-4 correspond to the IBV, S\&P, Vale, and BB series, respectively. The right side panels 1-4 correspond to the Merval, Itaú, Petro, and Brad series, respectively. sym., asym. = standardized symmetric and asymmetric Student $t$ innovations, respectively.

\begin{tabular}{|c|c|c|c|c|c|c|c|c|}
\hline \multirow{3}{*}{ Criterion } & \multicolumn{4}{|c|}{ Left panel } & \multicolumn{4}{|c|}{ Right panel } \\
\hline & \multicolumn{2}{|c|}{ Leverage } & \multicolumn{2}{|c|}{ Innovation } & \multicolumn{2}{|c|}{ Leverage } & \multicolumn{2}{|c|}{ Innovation } \\
\hline & without & with & sym. & asym. & without & with & sym. & asym. \\
\hline AIC & 0.00 & 100.0 & 0.00 & 100.0 & 0.00 & 100.0 & 10.20 & 89.80 \\
\hline $\mathrm{BIC}$ & 0.00 & 100.0 & 5.58 & 94.42 & 59.90 & 40.10 & 52.55 & 47.45 \\
\hline HQ & 0.00 & 100.0 & 0.00 & 100.0 & 23.35 & 76.65 & 23.47 & 76.53 \\
\hline AIC & 0.00 & 100.0 & 0.00 & 100.0 & 0.00 & 100.0 & 0.00 & 100.0 \\
\hline $\mathrm{BIC}$ & 0.00 & 100.0 & 10.95 & 89.05 & 0.00 & 100.0 & 56.85 & 43.15 \\
\hline HQ & 0.00 & 100.0 & 2.99 & 97.01 & 0.00 & 100.0 & 12.69 & 87.31 \\
\hline AIC & 0.00 & 100.0 & 100.0 & 0.00 & 0.00 & 100.0 & 19.29 & 80.71 \\
\hline $\mathrm{BIC}$ & 21.32 & 78.68 & 100.0 & 0.00 & 0.00 & 100.0 & 100.0 & 0.00 \\
\hline HQ & 0.00 & 100.0 & 100.0 & 0.00 & 0.00 & 100.0 & 77.16 & 22.84 \\
\hline AIC & 21.32 & 78.68 & 35.03 & 64.97 & 0.00 & 100.0 & 6.09 & 93.91 \\
\hline BIC & 41.12 & 58.88 & 100.0 & 0.00 & 0.00 & 100.0 & 56.35 & 43.65 \\
\hline HQ & 29.95 & 70.05 & 52.28 & 47.72 & 0.00 & 100.0 & 31.47 & 68.53 \\
\hline
\end{tabular}

Table 3 - Percentage of times the skewness parameter of the asymmetric Student $t$ distribution were significant at the $5 \%$ level.

\begin{tabular}{|ccccccccc|}
\hline & IBV & Merval & S\&P & Itaú & Vale & Petro & BB & Brad. \\
\hline GARCH & 99.49 & 77.04 & 92.54 & 99.49 & 0.00 & 11.17 & 57.87 & 72.08 \\
GJR & 100.0 & 79.08 & 99.50 & 89.34 & 0.00 & 34.52 & 44.16 & 68.53 \\
EGARCH & 100.0 & 72.45 & 99.00 & 77.66 & 0.00 & 9.14 & 51.78 & 68.53 \\
TGARCH & 100.0 & 72.96 & 99.00 & 74.62 & 0.00 & 14.21 & 49.24 & 68.53 \\
\hline
\end{tabular}

Table 4 - Percentage of times the leverage parameter of the GJR model was significant at the $5 \%$ level.

\begin{tabular}{|ccccccccc|}
\hline Distr. & IBV & Merval & S\&P & Itaú & Vale & Petro & BB & Brad. \\
\hline sym. & 100.0 & 79.70 & 100.0 & 100.0 & 98.98 & 100.0 & 68.02 & 100.0 \\
asym. & 100.0 & 82.74 & 100.0 & 100.0 & 95.94 & 100.0 & 66.50 & 100.0 \\
\hline
\end{tabular}

Table 5 presents the percentage of cases with loss larger than the one-step-ahead 95\%-VaR and $99 \%$-VaR. A good model should give a percentage close to the nominal value. It is preferred to have percentage smaller than larger that the nominal values. A good model should also have a large $p$-value for the LR test. The main conclusions are: 

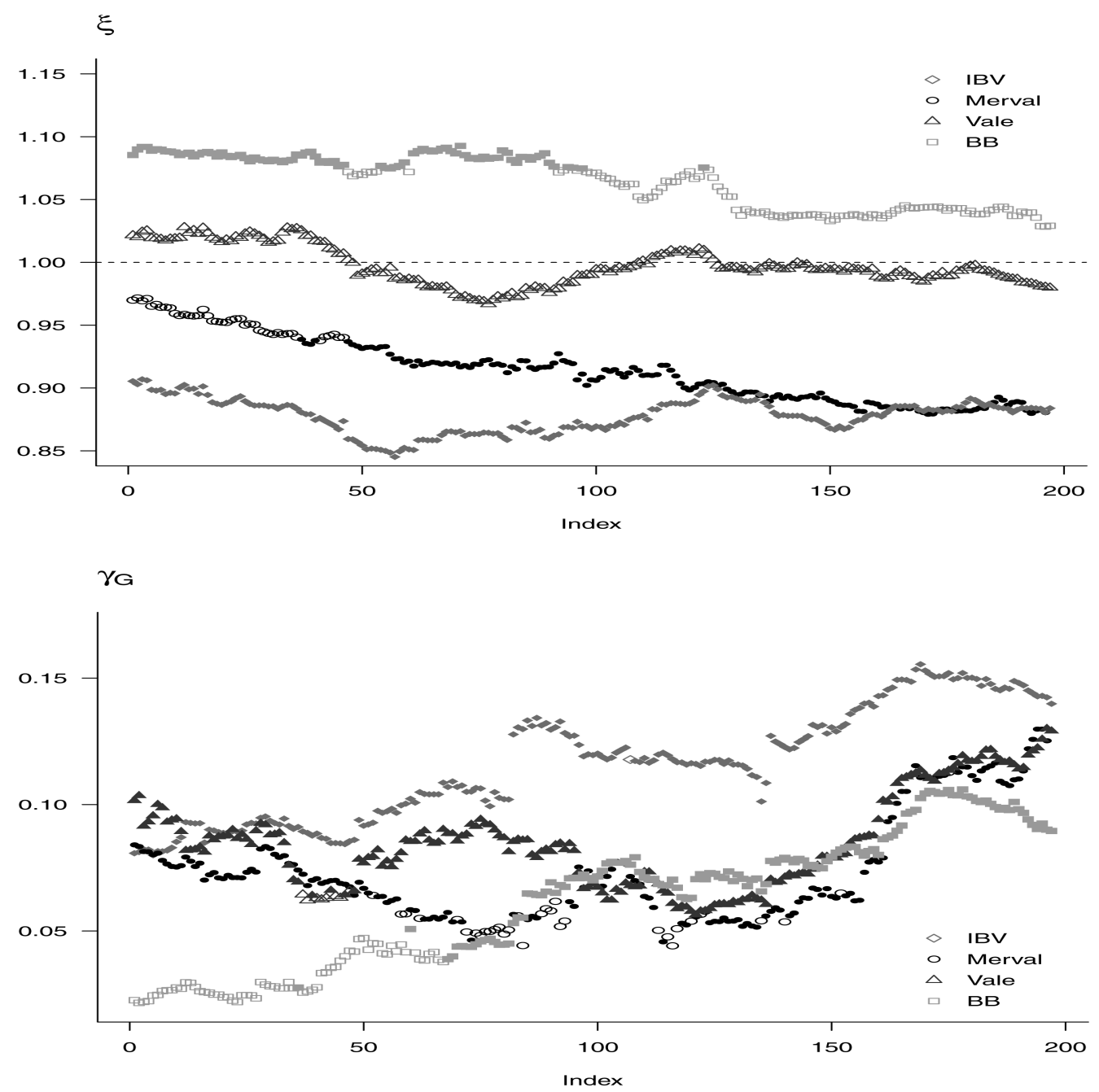

Figure 1 - Estimates of the asymmetry parameter of the error distributions $(\xi)$ and of the leverage parameter $\left(\gamma_{G}\right)$ of the GJR-GARCH model for the IBV, Merval, Vale, and BB series. Full symbols mean rejection of the null hypothesis at $5 \%$. Under the null hypothesis of no asymmetry, one has $(\xi=1)$; and under the null hypothesis of no leverage effect, $\left(\gamma_{G}=0\right)$.

- There is no meaningful difference in terms of percentage, although the models with asymmetric distributions are generally slightly better.

- For the $99 \%$-VaR, the models with asymmetric error distribution, except for S\&P series (for EGARCH and TGARCH), pass the LR test, with the smallest $p$-value equal to 0.15 . When we consider the symmetric error distribution, all the models fail for the IBV and $\mathrm{S} \& \mathrm{P}$ series. 

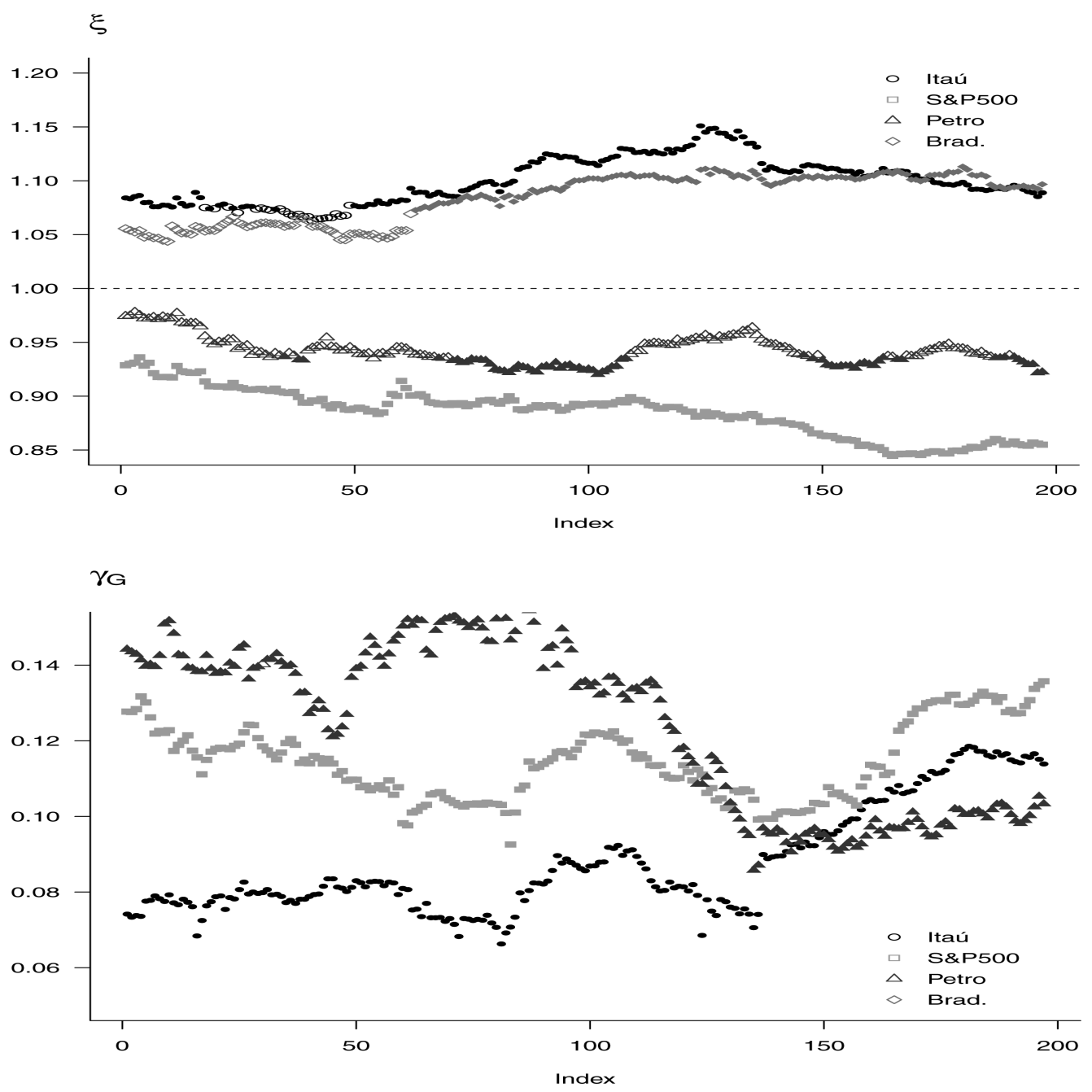

Figure 2 - Estimates of the asymmetry parameter of the error distributions $(\xi)$ and of the leverage parameter $\left(\gamma_{G}\right)$ of the GJR-GARCH model for the Itaú, S\&P, Petro and Brad series. Full symbols mean rejection of the null hypothesis at $5 \%$. Under the null hypothesis of no asymmetry, one has $(\xi=1)$; and under the null hypothesis of no leverage effect, $\left(\gamma_{G}=0\right)$.

- For the 95\%-VaR, the models with asymmetric error distribution, except for the Vale, Petro (for GARCH model), and S\&P series, pass the LR test at the 5\% level. When we consider the symmetric error distribution, all the models fail for the IBV, Merval, S\&P, Vale (except for GJR), and Petro series.

Considering the three methods of comparison we can say that the two stylized facts are present in most of the series analyzed, and that models taking into account these two stylized facts improve the estimation of the VaR. 
Table 5 - Percentage of cases with loss larger than the VaR and the $p$-value of the LR test for the conditional 95\%-VaR and 99\%-VaR. Panels 1-8 correspond to the IBV, Merval, S\&P, Itaú, Vale, Petro, BB, and Brad series, respectively.

\begin{tabular}{|c|c|c|c|c|c|c|c|c|}
\hline \multirow{3}{*}{ Model } & \multicolumn{4}{|c|}{ VaR 95\% } & \multicolumn{4}{|c|}{ VaR 99\% } \\
\hline & \multicolumn{2}{|c|}{ Percentage } & \multicolumn{2}{|c|}{$p$-value } & \multicolumn{2}{|c|}{ Percentage } & \multicolumn{2}{|c|}{$p$-value } \\
\hline & sym. & asym. & sym. & asym. & sym. & asym. & sym. & asym. \\
\hline GARCH & 93.10 & 94.11 & 0.032 & 0.447 & 98.27 & 98.98 & 0.086 & 0.901 \\
\hline GJR & 93.10 & 93.91 & 0.023 & 0.293 & 98.27 & 98.88 & 0.086 & 0.827 \\
\hline EGARCH & 92.79 & 93.81 & 0.006 & 0.144 & 98.07 & 98.68 & 0.024 & 0.529 \\
\hline TGARCH & 92.99 & 94.11 & 0.015 & 0.447 & 98.07 & 98.98 & 0.0235 & 0.901 \\
\hline GARCH & 92.96 & 93.47 & 0.022 & 0.118 & 98.47 & 98.61 & 0.245 & 0.545 \\
\hline GJR & 93.06 & 93.88 & 0.023 & 0.292 & 98.37 & 98.89 & 0.191 & 0.629 \\
\hline EGARCH & 92.76 & 93.67 & 0.010 & 0.200 & 98.16 & 98.77 & 0.1463 & 0.474 \\
\hline TGARCH & 92.96 & 93.67 & 0.022 & 0.088 & 98.27 & 98.77 & 0.186 & 0.474 \\
\hline GARCH & 92.84 & 93.23 & 0.002 & 0.025 & 97.61 & 98.61 & 0.001 & 0.287 \\
\hline GJR & 92.94 & 93.53 & 0.001 & 0.032 & 97.81 & 98.47 & 0.002 & 0.247 \\
\hline EGARCH & 91.74 & 92.74 & $<0.001$ & 0.012 & 96.82 & 97.91 & $<0.001$ & 0.002 \\
\hline TGARCH & 91.84 & 92.94 & $<0.001$ & 0.020 & 96.92 & 98.31 & $<0.001$ & 0.022 \\
\hline GARCH & 94.42 & 93.50 & 0.561 & 0.097 & 99.39 & 98.68 & 0.399 & 0.529 \\
\hline GJR & 94.92 & 93.81 & 0.928 & 0.229 & 99.19 & 98.98 & 0.776 & 0.901 \\
\hline EGARCH & 94.31 & 93.50 & 0.473 & 0.097 & 99.29 & 99.09 & 0.599 & 0.886 \\
\hline TGARCH & 94.62 & 93.60 & 0.737 & 0.132 & 99.29 & 99.19 & 0.599 & 0.776 \\
\hline GARCH & 93.20 & 93.20 & 0.046 & 0.046 & 98.48 & 98.48 & 0.246 & 0.246 \\
\hline GJR & 93.50 & 93.50 & 0.118 & 0.118 & 98.38 & 98.38 & 0.150 & 0.150 \\
\hline EGARCH & 93.10 & 93.20 & 0.023 & 0.034 & 98.38 & 98.38 & 0.150 & 0.150 \\
\hline TGARCH & 92.89 & 92.99 & 0.010 & 0.015 & 98.48 & 98.58 & 0.246 & 0.374 \\
\hline GARCH & 92.99 & 93.10 & 0.022 & 0.032 & 98.78 & 98.78 & 0.691 & 0.691 \\
\hline GJR & 93.30 & 93.91 & 0.026 & 0.071 & 98.48 & 98.68 & 0.246 & 0.529 \\
\hline EGARCH & 93.10 & 93.50 & 0.023 & 0.097 & 98.58 & 98.68 & 0.374 & 0.529 \\
\hline TGARCH & 93.30 & 93.50 & 0.026 & 0.055 & 98.68 & 98.68 & 0.529 & 0.529 \\
\hline GARCH & 95.63 & 95.23 & 0.235 & 0.080 & 98.88 & 98.58 & 0.827 & 0.374 \\
\hline GJR & 95.74 & 95.43 & 0.062 & 0.149 & 98.88 & 98.68 & 0.827 & 0.529 \\
\hline EGARCH & 95.74 & 95.33 & 0.381 & 0.436 & 98.88 & 98.48 & 0.827 & 0.246 \\
\hline TGARCH & 95.74 & 95.33 & 0.381 & 0.436 & 98.98 & 98.58 & 0.901 & 0.374 \\
\hline GARCH & 95.23 & 94.42 & 0.591 & 0.561 & 99.29 & 99.19 & 0.599 & 0.776 \\
\hline GJR & 95.33 & 94.72 & 0.588 & 0.415 & 99.09 & 98.98 & 0.886 & 0.901 \\
\hline EGARCH & 95.13 & 94.62 & 0.084 & 0.360 & 99.09 & 98.88 & 0.886 & 0.827 \\
\hline TGARCH & 95.13 & 94.62 & 0.084 & 0.360 & 99.19 & 98.88 & 0.776 & 0.827 \\
\hline
\end{tabular}

\section{CONCLUDING REMARKS}

In this paper we analyzed eight series in order to test whether two stylized facts are present: asymmetry in the error distributions and the leverage effect. We first compared the models using 
the AIC, BIC, and HQ information criteria, and by using hypothesis testing. In both methods, we found evidence that the two stylized facts are present in most of the series analyzed. In the third method, we compared the VaR estimates and found that in VaR estimation, the models with asymmetric errors performed much better than those with symmetric distributions, in terms of the LR test.

\section{ACKNOWLEDGMENTS}

This work was partially supported through grants 2008/51097-6 and 2011/02881-9, São Paulo Research Foundation (FAPESP), and grants from CAPES and CNPq.

\section{REFERENCES}

[1] AKAIKE H. 1974. A new look at the statistical model identification. IEEE Transactions on Automatic Control, 19: 716-722.

[2] BLACK R. 1976. Studies in stock price volatility changes. Proceedings of the 1976 Meeting of the American Statistical Association, Business and Economics Statistics Section, 177-181.

[3] BollersleV T. 1986. Generalized autoregressive conditional heteroskedasticity. Journal of Econometrics, 31: 307-327.

[4] Christoffersen P. 1998. Evaluating interval forecasts. International Economic Review, 39: 841862.

[5] Ding Z, Engle RF \& GRAnger CWJ. 1993. A long memory property of stock market return and a new model. Journal of Empirical Finance, 1: 83-106.

[6] EHLERS RS. 2012. Computational tools for comparing asymmetric GARCH models via Bayes factors. Mathematics and Computers in Simulation, 82: 858-867.

[7] FernándeZ C \& STEel M. 1998. On Bayesian modelling of fat tails and skewness. Journal of the American Statistical Association, 93: 359-371.

[8] French KG, Schwert W \& Stambaugh RF. 1987. Expected stock returns and volatility. Journal of Financial Economics, 19: 3-29.

[9] Glosten LR, Jagannathan R \& RunkLe DE. 1993. On the relation between the expected value and the volatility of the nominal excess return on stocks. Journal of Finance, 48: 1779-1801.

[10] Kon S. 1982. Models of stock returns, a comparison. Journal of Finance, 39: 147-165.

[11] HANNAN EJ \& QUinN BG. 1979. The determination of the order of an autoregression. Journal of the Royal Statistical Society B, 41: 190-195.

[12] HAnsen B. 1994. Autoregressive conditional density estimation. International Economic Review, 35: 705-730.

[13] Hents Chel L. 1995. All in the family nesting symmetric and asymmetric GARCH models. Journal of Financial Economics, 39: 71-104.

[14] LAMBert P \& LAUREnT S. 2001. Modelling financial time series using GARCH-type models and a skewed Student density. Discussion paper 0125, Institut de Statistique, Université Catholique de Louvain, Louvain-la-Neuve, Belgium. 
[15] NeLSON DB. 1991. Conditional heteroskedasticity in asset returns: A new approach. Econometrica, 59: $347-370$.

[16] RodrígUez MJ \& RUIZ E. 2012. Revisiting several popular GARCH models with leverage effect: Differences and similarities. Journal of Financial Econometrics, 10: 637-668.

[17] SCHWARZ GE. 1978. Estimating the dimension of a model. Annals of Statistics, 6: 461-464.

[18] Simkowitz M \& Beedles W. 1980. Asymmetric stable distributed security returns. Journal of the American Statistical Association, 75: 306-312.

[19] ZAKOÏAN JM. 1994. Threshold heteroskedastic models. Journal of Economic Dynamics and Control, 18: $931-955$. 